\title{
Quadrature formula for evaluating left bounded Hadamard type hypersingular integrals
}

\begin{abstract}
Left semi-bounded Hadamard type Hypersingular integral (HSI) of the form $\mathrm{H}(\mathrm{h}, \mathrm{x})=1 /^{\prime} 1+\mathrm{x} / 1-\mathrm{x} \approx \mathrm{s} * * 11-\mathrm{t} / 1+\mathrm{t} \mathrm{h}(\mathrm{t})(\mathrm{t}-\mathrm{x}) 2 \mathrm{dt}, \mathrm{x} \in(-1.1)$, Where $\mathrm{h}(\mathrm{t})$ is a smooth function is considered. The automatic quadrature scheme (AQS) is constructed by approximating the density function $h(t)$ by the truncated Chebyshev polynomials of the fourth kind. Numerical results revealed that the proposed AQS is highly accurate when $h(t)$ is choosing to be the polynomial and rational functions. The results are in line with the theoretical findings.
\end{abstract}

Keyword: AQS; Chebyshev polynomials; Hypersingular integral; Left semi-bounded 